\title{
Anti-proliferative effect of honokiol in oral squamous cancer through the regulation of specificity protein 1
}

\author{
DONG-WOOK KIM ${ }^{1 *}$, SEON MI KO ${ }^{1 *}$, YOUNG-JOO JEON ${ }^{1 *}$, YOUNG-WOOCK NOH ${ }^{2}$, NAG-JIN CHOI ${ }^{3}$, \\ SUNG-DAE $\mathrm{CHO}^{4}$, HONG SEOP MOON ${ }^{5}$, YOUNG SIK CHO ${ }^{6}$, JAE-CHEN SHIN ${ }^{7,8}$, SEON-MIN PARK ${ }^{7}$, \\ KANG SEOK SEO ${ }^{9}$, JI-YOUNG CHOI ${ }^{10}$, JUNG-IL CHAE ${ }^{1}$ and JUNG-HYUN SHIM ${ }^{5}$
}

\author{
${ }^{1}$ Department of Oral Pharmacology, School of Dentistry and Institute of Dental Bioscience, BK21 project, \\ Chonbuk National University, Jeonju; ${ }^{2}$ Graduate School and Department of Analytical Science and Technology, \\ Chungnam National University, Daejeon; ${ }^{3}$ Department of Animal Science, College of Agricultural and Life Science, \\ ${ }^{4}$ Department of Oral Pathology, Chonbuk National University, Jeonju; ${ }^{5}$ College of Pharmacy, Mokpo National University, \\ Jeonnam; ${ }^{6}$ Department of Pharmacy, Keimyung University, Daegu; ${ }^{7}$ Pohang Center for Evaluation of Biomaterials, Pohang, \\ Gyeongbuk; ${ }^{8}$ Department of Life Science, Division of Molecular and Life Science, Pohang University of Science \\ and Technology, Pohang, Gyeongbuk; ${ }^{9}$ Department of Animal Science and Technology, Sunchon National \\ University, Suncheon; ${ }^{10}$ Medical Proteomics Research Center, KRIBB, Daejeon, Republic of Korea
}

Received March 27, 2013; Accepted May 16, 2013

DOI: 10.3892/ijo.2013.2028

\begin{abstract}
Honokiol (HK), a novel plant-derived natural product, is a physiologically activated compound with polyphenolic structure, and has been identified to function as an anticancer agent. It has been widely used in several diseases as a traditional medicine for a long time. We investigated whether HK could show anticancer effects on two oral squamous cell lines (OSCCs), HN-22 and HSC-4. We demonstrated that HK-treated cells showed dramatic reduction in cell growth and apoptotic cell morphologies. Intriguingly, the transcription factor specificity protein 1 ( $\mathrm{Sp} 1)$ was significantly inhibited by HK in a dose-dependent manner. Furthermore, we checked changes in cell cycle regulatory proteins and anti-apoptotic proteins at the molecular level, which are known as Sp1 target genes. The important key regulators in the cell cycle such as p27 and p21 were up-regulated by HK-mediated down-regulation of Sp1,
\end{abstract}

Correspondence to: Professor Jung-Il Chae, Department of Oral Pharmacology, School of Dentistry, Brain Korea 21 Project, Chonbuk National University, Jeonju 561-756, Republic of Korea

E-mail: jichae@jbnu.ac.kr

Professor Jung-Hyun Shim, Department of Pharmacy, College of Pharmacy, Mokpo National University, 1666 Youngsan-ro, Muan-gun, Jeonnam 534-729, Republic of Korea

E-mail: s1004jh@gmail.com

*Contributed equally

Abbreviations: MTS, 3-(4,5-dimethylthiazol-2-yl)-5-(3-carboxyme thoxyphenyl)-2-(4-sulfophenyl)-2H-tetrazolium; PMS, phenazine methosulfate; DAPI, 4'-6-diamidino-2-phenylindole; TUNEL, terminal deoxynucleotidyltransferase UTP nick end labeling

Key words: honokiol, oral squamous cancer cell, specificity protein 1, apoptosis whereas anti-apoptotic proteins including Mcl-1 and survivin were decreased, resulting in caspase-dependent apoptosis. Taken together, results from this study suggest that HK could modulate Sp1 transactivation and induce apoptotic cell death through the regulation of cell cycle and suppression of anti-apoptotic proteins. In addition, HK may be used in cancer prevention and therapies to improve the clinical outcome as an anticancer drug.

\section{Introduction}

Oral squamous cell carcinoma (OSCC) is a common type of malignant tumor in the world. New cases of oral cancer occur at around 275,000 patients per year, OSCC cases comprise approximately $>90 \%$ of diagnosed patients with oral cancer (1). Although conventional treatments of oral cancer, including surgery, radiation and chemotherapy, have well advanced to date, the five-year survival rate remains $<50 \%$ (2). Hence, discovery and development of effective chemotherapeutic agents for OSCC might result in improved survival rate of OSCC patients.

Natural products as sources of new drugs have been explored and expanded in anticancer drug development for the past several decades. In fact, $74.8 \%$ of all the anticancer drugs have been discovered and semi-modified from natural sources, for example, 20 small molecules were approved in 2010 (3). Among the plant-derived products, honokiol (HK) is the most attractive natural compound since it has been widely used to treat several diseases, including stroke, anxiety, fever and ischemic heart disease, by Chinese (houpo) and Japanese (saiboku-to) as a traditional herbal medicine (4). HK is a polyphenolic compound containing physiologically active small molecules, it is isolated from the cones, bark and leaves of Magnolia species (Magnolia officinalis or grandiflora) $(4,5)$.

Many researchers have paid attention to biological effects of $\mathrm{HK}$ in various cancer cells because it has many remarkable 
pharmacological abilities, such as anti-inflammatory, antithrombotic, anti-arrhythmic, anti-platelet and anti-oxidative effects, without appreciable toxicity (6-9). Several studies on the effect of HK demonstrated its anticancer activity in various cancer cell lines and tumor models (10-14). HK was first proposed as a potent chemotherapy candidate for cancer therapy due to its antitumor activity against xenografted tumors in mice (15). In that study, HK treatment resulted in inhibition of tumor growth rate up to $50 \%$. In addition, it was found to induce apoptotic cell death in B cell chronic lympocytic leukemia cell lines through a caspase-dependent pathway. Furthermore, combinatorial treatments of a low dose HK with chemotherapeutic agents such as fludarabine, cladribine and chlorambucil, enhanced the cytotoxic effect (10). HK has also been shown to inhibit the NF- $\mathrm{kB}$ signaling pathway via decreasing the TNF- $\alpha$-induced NF- $\kappa B$ activation, IKK activity,

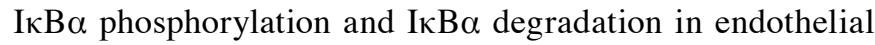
cells, monocytes, breast cancer and cervical cancer (16-18). Consequently, HK has received attention as a potent anticancer drug due to its ability in the regulation of multiple signal transductions in various cell lines.

Although the anticancer effects of HK have been well demonstrated against numerous cancer cell lines and models, little is known about the effect of HK on oral squamous cell carcinoma (OSCC). To characterize the effect of HK on OSCC, this study specifically examined the anticancer effect of HK on cell viability against two oral squamous cell carcinoma cell lines, $\mathrm{HN}-22$ and HSC-4, and identified the regulated proteins by HK treatment in these cells. Interestingly, an important gene regulating protein specificity protein $1(\mathrm{Spl})$ in cell proliferation, cell cycle progression and oncogenesis was significantly regulated when cells were treated with HK (19). Subsequently, we explored whether downstream proteins of Sp1 and key apoptotic proteins could be affected in their expression toward apoptotic cell death through alteration of Sp1 expression by HK treatment. Our results provide insight for the chemotherapeutic efficacy of HK in oral squamous cells.

\section{Materials and methods}

Cell culture and reagents. The human oral squamous cancer cells, HN-22 and HSC-4, were generously provided by Dr Sung-Dae Cho (Chonbuk National University, Jeonju, Korea) and cultured in Hyclone Dulbecco's modified Eagle's medium (DMEM; Thermo Scientific, Logan, UT) containing $10 \%$ heat-inactivated fetal bovine serum and $100 \mathrm{U} / \mathrm{ml}$ each of penicillin and streptomycin (Thermo Scientific) at $37^{\circ} \mathrm{C}$ with $5 \% \mathrm{CO}_{2}$ in humidified air. $\mathrm{HK}$ was purchased from Sigma-Aldrich (St. Louis, MO).

Cell viability assay. Cell viability was measured using the CellTiter 96 ${ }^{\mathrm{TM}}$ AQueous assay kit (Promega, Madison, WI) according to the manufacturer's protocol. Both $\mathrm{HN}-22$ and HSC-4 cells were seeded on a 96-well microtiter plate (HN-22, $2 \times 10^{3}$ cells/well and HSC- $4,3 \times 10^{3}$ cells/well) and then cells were treated with different doses of $0,2.5,5$ or $10 \mu \mathrm{g} / \mathrm{ml} \mathrm{HK}$. Cell viability was measured by adding dehydrogenase enzyme substrate (MTS, 3-(4,5-dimethylthiazol-2-yl)-5-(3-carboxymethoxyphenyl)-2-(4-sulfophenyl)-2H-tetrazolium) and electron coupling reagent (PMS, phenazinemethosulfate) and then plates were incubated at $37^{\circ} \mathrm{C}$ in $5 \% \mathrm{CO}_{2}$ for $2 \mathrm{~h}$ after $24 \mathrm{~h}$ post-treatment of HK. The absorbance was measured at $490 \mathrm{~nm}$ using GloMax-Multi Microplate Multimode Reader (Promega). Percentages of cell viabilities of HK treated cells were normalized to that of untreated cells.

Sp1 knockdown using siRNA. The endogenous Sp1 knockdown was induced via the transient transfection of siRNA. Knockdown of Spl was performed using a pool of four duplexes targeting Sp1 (TARGETplus SMARTpool siRNA, Thermo Scientific Dharmacon, Lafayette, CO). HN-22 and HSC-4 cells were seeded in 96-well plates and $100-\mathrm{mm}$ culture dishes, and Sp1 targeting siRNA or non-targeting controls (Dharmacon) at a $50 \mathrm{nM}$ were introduced using the DharmaFECT2 transfection reagent. After transfection, cells were subjected to MTS assay and western blot analysis.

Terminal deoxynucleotidyl transferase-mediated dUTP nick end labeling assay. The apoptotic events were visualized by terminal deoxynucleotidyltransferase UTP nick end labeling (TUNEL) assay using an in situ cell death detection kit (Roche, Mannheim, Germany). Cells were prepared as described previously in 6-well plates with coverslips. After HK administration, a TUNEL assay was performed with an In Situ Cell Death Detection kit according to the manufacturer's manual. In brief, cells were fixed and permeabilized with cytofix/cytoperm solution (BD Biosciences, San Diego, CA) for $30 \mathrm{~min}$. The fixed and permeabilized cells were then treated with the TUNEL reaction mixture and incubated in a humidified dark chamber at $37^{\circ} \mathrm{C}$ for $1 \mathrm{~h}$. The samples were washed with PBS and then the stained cells were observed under a FluoView confocal laser microscope.

Immunocytochemistry. The cells were seeded over each sterilized glass coverslips on 6-well tissue culture plates for $24 \mathrm{~h}$ and incubated with HK for $48 \mathrm{~h}$. The cells were fixed and permeabilized with cytofix/cytoperm solution for $30 \mathrm{~min}$. For expression of Sp1, the cells were blocked with $1 \%$ BSA and then incubated with monoclonal $\mathrm{Sp} 1$ antibody at $4^{\circ} \mathrm{C}$ overnight. After washing with PBS containing 0.05\% Tween-20 (PBST) solution, the Sp1 antibody was reacted with a Jackson 488-conjugated anti-mouse secondary antibody at room temperature for $1 \mathrm{~h}$ and mounted with Mountin solution-Vectashield mounting medium for fluorescence with DAPI (Vector Laboratories Inc., Burlingame, CA) onto the cells. The cells were visualized using a FluoView confocal laser microscope.

Western blot analysis. The total cell lysate were prepared using PRO-PREP ${ }^{\mathrm{TM}}$ protein Extraction Solution (iNtRON Biotechnology, Seoul, Korea) containing $1 \mu \mathrm{g} / \mathrm{ml}$ aprotinin, $1 \mu \mathrm{g} / \mathrm{ml}$ leupeptin and $1 \mu \mathrm{M}$ PMSF. Equal amounts of total protein were separated on 10 or $15 \% \mathrm{v} / \mathrm{v}$ SDS-PAGE and then transferred onto polyvinylidenedifluoride (PVDF) membranes. After blocking with 5\% non-fat dried milk in PBST containing $0.1 \%$ Tween-20, membranes were immunoblotted with specific primary antibodies against Sp1 (1C6), p21 (F-5), p27 (C-19), cyclin D1 (M-20) (Santa Cruz Biotechnology, Santa Cruz, CA), PARP (BD Biosciences), Mcl-1, survivin, cleaved-caspase-3 (Cell Signaling, Danvers, MA) and $\beta$-actin (AC-74) (Sigma-Aldrich) overnight at $4^{\circ} \mathrm{C}$. After washing with PBST, 
A

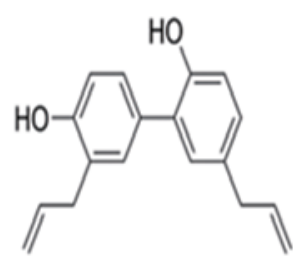

Honokiol (HK)
B

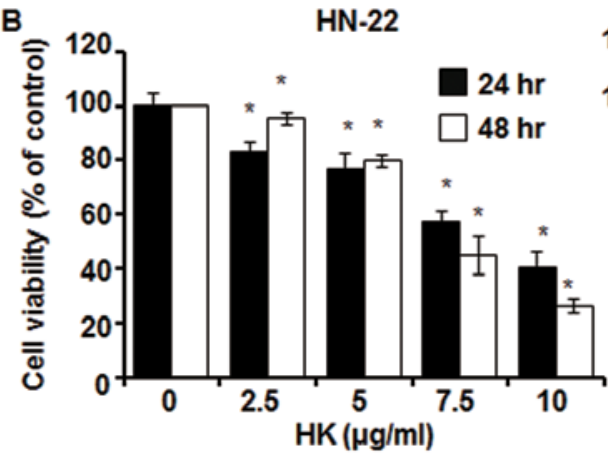

HSC-4

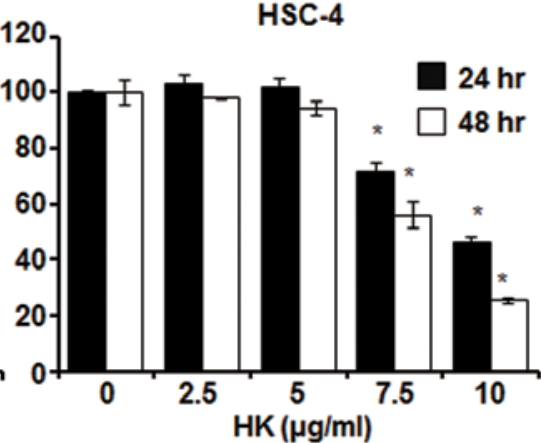

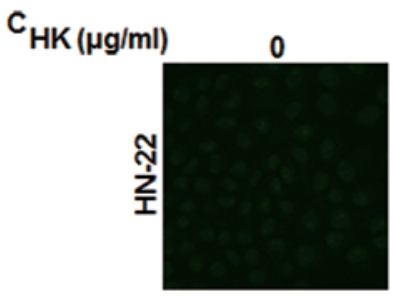
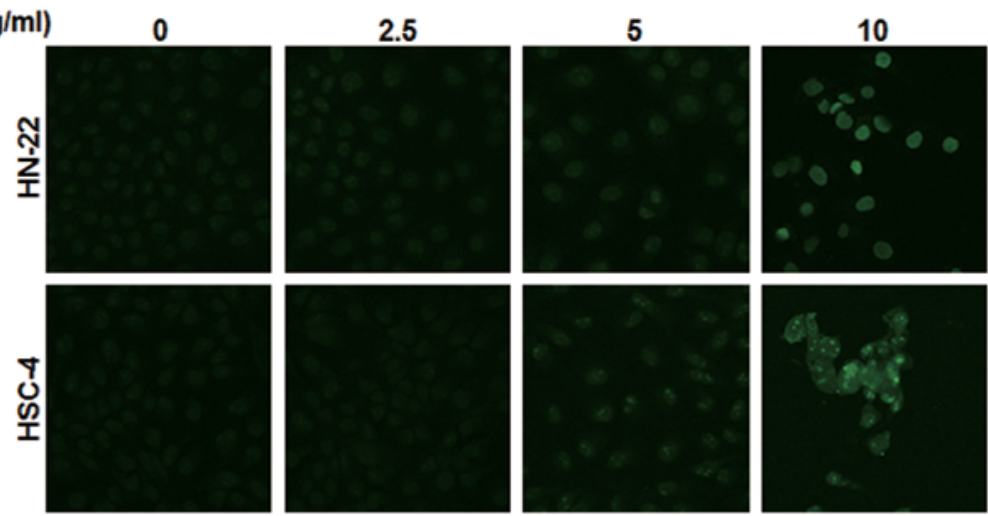

Figure 1. The effect of honokiol (HK) on cell viability of oral cancer cells. (A) Chemical structure of HK. (B) The cell viability effect of HK on HN-22 and HSC-4

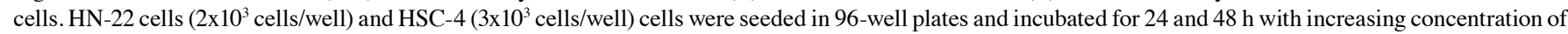
HK in 10\% FBS-DMEM. Cell viability was estimated using an MTS assay kit, as described in Materials and methods. Results are indicated as cell viability relative to the HK untreated, and data are the means \pm SD from three independent experiments. The asterisk indicates a significant difference compared to the negative control (untreated cells) $\left({ }^{*} \mathrm{p}<0.05\right)$. (C) TUNEL analyses were performed using a FluoView confocal laser microscope. The morphological changes observed in the HK treated $(2.5,5$ and $10 \mu \mathrm{g} / \mathrm{ml})$ or untreated $\mathrm{HN}-22$ and HSC-4 cells at $48 \mathrm{~h}$ post-treatment.

the membranes were incubated with horseradish-peroxidaseconjugated anti-mouse IgG or anti-rabbit IgG (Santa Cruz Biotechnology) and chemiluminescence signals were enhanced by Pierce ECL Western Blotting Substrate (Thermo Scientific, Rockford, IL).

Statistical analysis. Statistical significance was assessed using a Student's t-test. A p-value of $<0.05$ compared with the non-treated cool was considered statistically significant.

\section{Results}

Honokiol inhibits cell viability and induces apoptosis of OSCCs. Previously, it has been reported that HK inhibits cell proliferation and tumor growth of various cell lines derived from different cancers $(15,20,21)$. Therefore, we examined whether HK could effectively suppress the cell proliferative capability of the OSCCs, HN-22 and HSC-4. To determine the cell viability, we employed MTS assay after HK treatment with different concentrations $(2.5,5,7.5$ or $10 \mu \mathrm{g} / \mathrm{ml})$ and different time-points ( 24 or $48 \mathrm{~h}$ ) into HN-22 or HSC-4. The $\mathrm{IC}_{50}$ of $\mathrm{HK}$ for $48 \mathrm{~h}$ treatment in the $\mathrm{HN}-22$ and HSC-4 cells was calculated to be approximately 7.1 and $8.0 \mu \mathrm{g} / \mathrm{ml}$ (Fig. 1B). The cell viability of $\mathrm{HN}-22$ was, respectively, $95.4 \pm 2.3,79.7 \pm 2.0,44.7 \pm 7.1$ and $26.3 \pm 2.3 \%$ at $2.5,5,7.5$ and $10 \mu \mathrm{g} / \mathrm{ml}$ of $\mathrm{HK}$ compared with the untreated control cells when viability was calculated at $48 \mathrm{~h}$ post-treatment. In the case of HSC-4, viability was $97.7 \pm 0.5$, $94.2 \pm 2.7,56.0 \pm 4.8$ and $25.2 \pm 0.9$ at $2.5,5,7.5$ and $10 \mu \mathrm{g} / \mathrm{ml}$, respectively, of $\mathrm{HK}$ compared to that of the untreated control cells at $48 \mathrm{~h}$ post-treatment. Next, we confirmed the induction of apoptosis by HK treatment. TUNEL assay was performed to visualize the cells undergoing apoptosis to detect DNA fragmentation that resulted from the apoptotic signaling cascade. As shown in Fig. 1C, TUNEL positive cells were markedly increased in high-dose treated $(10 \mu \mathrm{g} / \mathrm{ml})$ cells in both $\mathrm{HN}-22$ and HSC-4 in comparison to the untreated or low-dose treated cells. These data show that HK treatment effectively inhibited cell growth and led to apoptotic cell death in OSCCs.

Spl protein level is decreased by honokiol. The transcription factor of Sp1 highly overexpressed in various cancer-derived cell lines including human glioblastoma, lung and pancreatic cancers etc., and has regulated transcriptional activity on differentiation, growth and oncogenesis genes (e.g. cyclins, $c-m y c$ and p53) through modulation of target gene promoter $(19,22-$ 25). If the expression level of Sp1 protein could be effectively modulated by a chemotherapeutic agent, then the agent can be a potent candidate for an anticancer drug through suppression of tumor progression. Thus, to determine whether Sp1 protein levels were reduced by $\mathrm{HK}$ under the same conditions as MTS assays, two OSCC cell lines (HN-22 and HSC-4) were treated with different doses of $\mathrm{HK}$ at $0,2.5,5$ and $10 \mu \mathrm{g} / \mathrm{ml}$ for $48 \mathrm{~h}$. The 
A
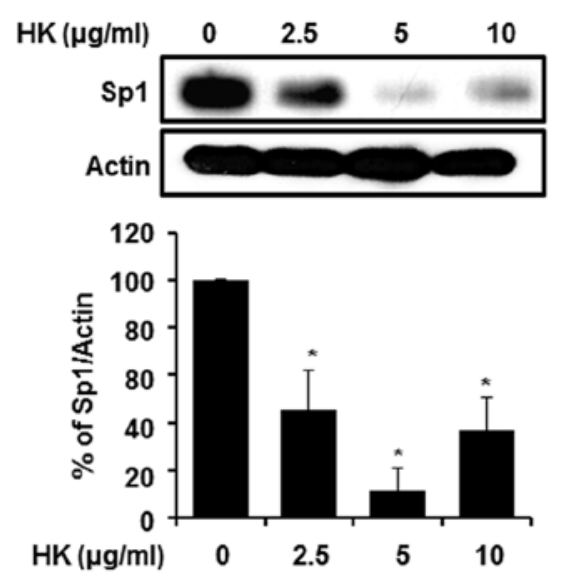

C

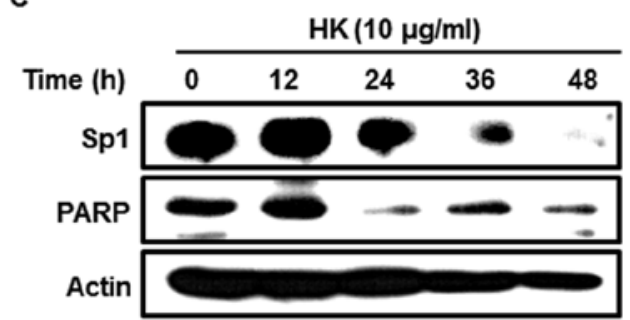

B
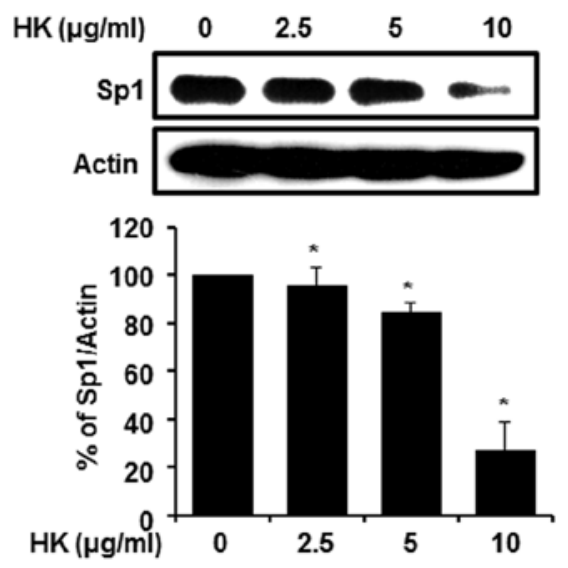

D

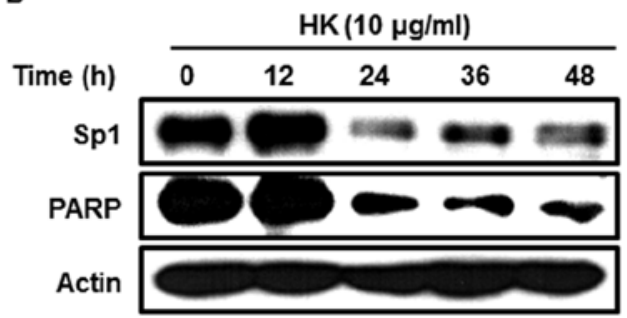

E

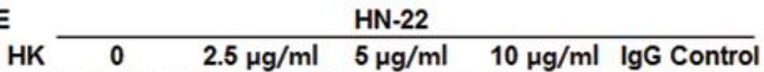

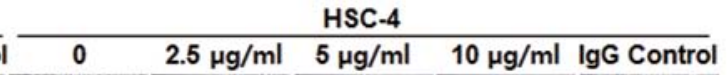

号
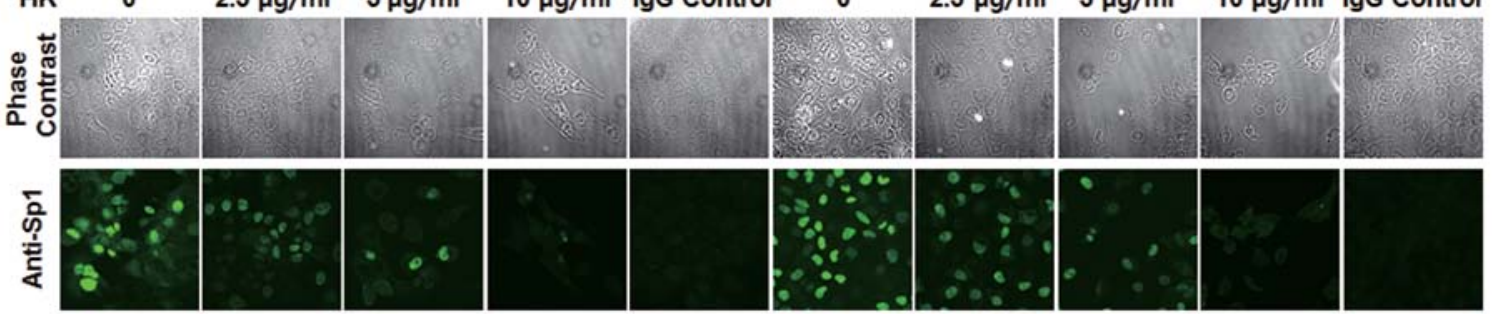

$\frac{\bar{n}}{8}$
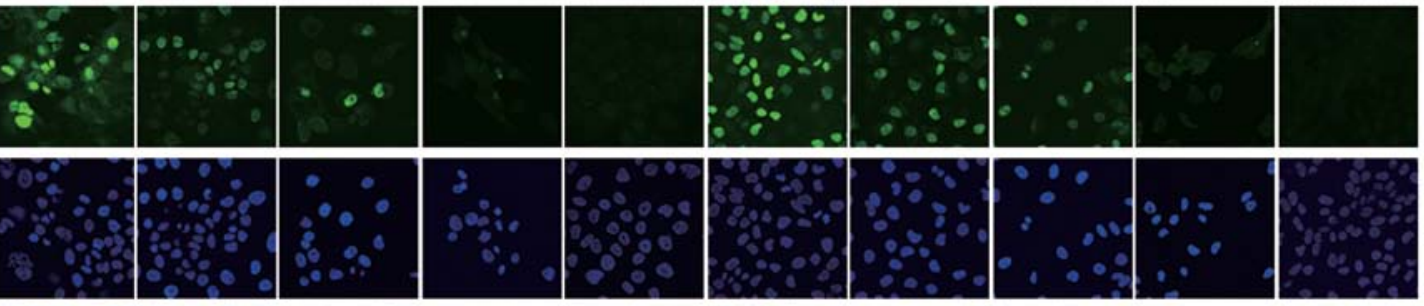

흔
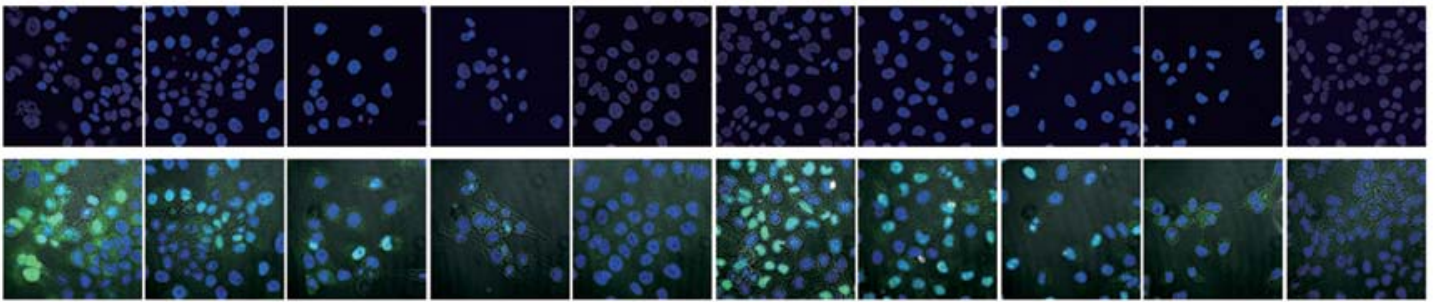

Figure 2. The effect of honokiol (HK) on specificity protein 1 (Sp1) protein expression in HN-22 and HSC-4 cells. The HN-22 and HSC-4 cells were incubated with different concentrations of $\mathrm{HK}$ for $48 \mathrm{~h}$. The cells were harvested and prepared for western blot analysis as described in Materials and methods. (A and B) Protein expression levels of Sp1 were detected using a specific antibody against Spl and its levels were quantified after actin normalization. The HK-treated cells were compared with the untreated cells, and data are the means \pm SD of three independent experiments. The asterisk indicates a significant difference compared with the negative control (untreated cells) ( $\left.{ }^{*} \mathrm{p}<0.05\right)$. Time-dependent effects of HK on Sp1 and PARP expressions were performed in (C) HN-22 and (D) HSC-4 cells for $48 \mathrm{~h}$ with 12-h intervals. (E) Immunocytochemistry analysis was performed in HK treated HN-22 and HSC-4 cells. HN-22 and HSC-4 cells were treated with different concentrations of HK for $48 \mathrm{~h}$ and cells were immunostained with Spl specific antibody, and then signals were detected with Jackson 488 -conjugated anti-mouse secondary antibody. DAPI was used for nucleus staining.

Sp1 levels were dramatically decreased in the treated cells with maximum $88.1 \pm 9.4 \%$ compared to the $\mathrm{HN}-22$ untreated group and $73.2 \pm 12.2 \%$ of the HSC-4 untreated group (Fig. $2 \mathrm{~A}$ and B). Consistent with these observations, immunocytochemical results also showed a decreased level of Sp1 positive cells in a dose-dependent manner in HN-22 and HSC-4 (Fig. 2E). To address the cellular effect of down-regulated Spl by HK, we examined the alterations of apoptotic-related protein, PARP, by western blot analysis along with $\mathrm{Sp} 1$ expression. When the expression levels of Sp1 and PARP were monitored for $48 \mathrm{~h}$ with 12-h intervals, Sp1 levels were dramatically diminished as time passed and full-length PARP also showed the parallel changes with Sp1 under HK treated conditions (Fig. 2C and D). These results collectively suggest that down-regulation of Sp1 by HK treatment could lead to apoptotic cell death.

Suppression of Spl expression leads to apoptotic cell death. It has been reported that transcriptional activity of $\mathrm{Sp} 1$ has an 
A

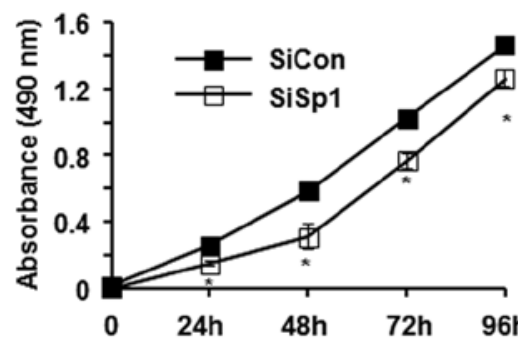

B

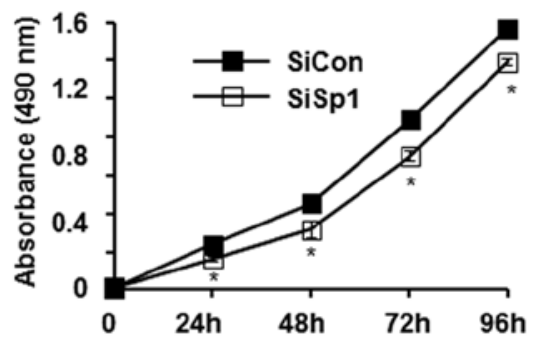

D

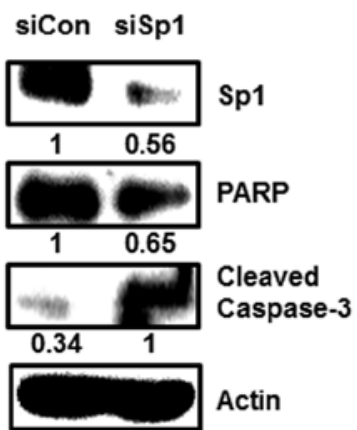

Figure 3. Knockdown of specificity protein 1 (Sp1) for functional study on cell proliferation of oral cancer cells. Sp1 specific (siSp1) or non-target siRNA (siCon) were transfected into the (A) HN-22 and (B) HSC-4 cells. Cell proliferation in siCon and siSpl-transfected (A) HN-22 and (B) HSC-4 cells was quantified by an MTS assay at 24, 48, 72 and $96 \mathrm{~h}$ post-transfection. Data are the means \pm SD of three independent experiments. The asterisk indicates a significant difference in the siSpl transfected cells compared with the siCon transfected cells ( $\left.{ }^{*} \mathrm{p}<0.05\right)$. The siCon or siSpl transfected (C) HN-22 and (D) HSC-4 cell lysates were determined by western blot analysis using anti-Sp1, anti-PARP and anti-cleaved-caspase-3. The values measured by Image J densitometry are representative of three independent experiments. Actin was used as a loading control.

important role in oncogenesis. Indeed, many cancer-derived cells showed enforced expression levels of Sp1 in comparison to normal cells. Moreover, the level of Spl was shown to affect the fate of cancer cells through modulating the genes involved in cell cycle progression, growth and apoptosis $(24,25)$. Our previous study reported that $\mathrm{Sp} 1$ down-regulated cancer cells were shown to decrease the proliferation rate $(26,27)$. Therefore, we investigated to clearly evaluate whether Sp1 expression level has an effect on cell viability and apoptosis in HN-22 and HSC-4 cells. To determine the cellular effect by $\mathrm{Sp1}$, we transiently transfected the $\mathrm{Sp} 1$ specific targeting siRNA (siSp1) into HN-22 and HSC-4 and then monitored cell viabilities at different transfection time points $(24,48,72$ and 96 h). As expected, siSp1 transfected HN-22 and HSC-4 showed reduced viabilities compared to siCon transfected $\mathrm{HN}-22$ and HSC-4 cells (Fig. 3A and B). Apoptosis inducing protein levels were also significantly changed in Sp1 knocked down cells by siSp1. The full-length PARP decreased according to the Sp1 expression level, whereas cleaved-caspase-3 increased (Fig. 3C and D). Our results demonstrate that the level of Sp1 expression plays an important role in the physiological progression of OSCCs (HN-22 and HSC-4).

HK treatment shows the same effects of Spl in suppressed conditions. To determine the regulatory role of $\mathrm{HK}$, we focused on the expression levels of the Spl downstream targets and pro-apoptotic proteins. We found that cell cycle arrest proteins, such as p27 and p21, were markedly enhanced in a dose-dependent manner by $\mathrm{HK}$, whereas cell proliferation and survival associated proteins, such as cyclin D1, Mcl-1 and survivin, were decreased by HK treatment (Fig. 4A and B). Moreover, when we tested pro-apoptotic protein levels at different doses, HK dose-dependently caused activation of caspase- 3 and PARP in OSCCs (Fig. 5A and B).

\section{Discussion}

$\mathrm{HK}$ is a physiologically activated natural product, which has been widely used in China and Japan as a traditional herbal medicine for treatment of stroke, fever, anxiety and nervous disturbance (5) (Fig. 1A). It was reported that HK has multifunctional roles in cellular processes (4). Several studies have reported that $\mathrm{HK}$ has an antitumor effect on various cancer derived cells, including B-CLL, prostate and hepatoma cell lines (10-13). Despite numerous studies on cancer cells, anticancer activities of HK on OSCCs are not well understood.

In this study, we extensively explored the apoptotic effects of HK in OSCCs, since oral cancer is one of serious diseases in many parts of the world. Oral cancer, which is the cancer of the oral and pharyngeal cavities, is ranked as the sixth most commonly occurring cancer in the world. Oral squamous cell carcinomas (OSCCs) account for over $90 \%$ of oral cancers (1). Despite several clinical approaches including surgical resection, radiation therapy, chemotherapy or their combinations, OSCCs still have lower survival rates as well as being the most aggressive malignant tumor type. Thus, efficacious drugs are highly required for OSCCs treatment. In HK treatment of the two OSCCs, HN-22 and HSC-4, at different times and concentrations (Fig. 1B), TUNEL positive cells were increased in a dose-dependent manner (Fig. 1C). 


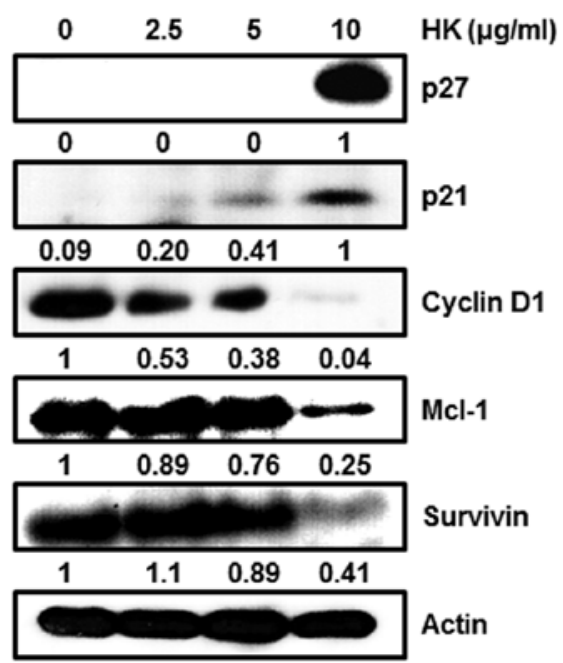

B

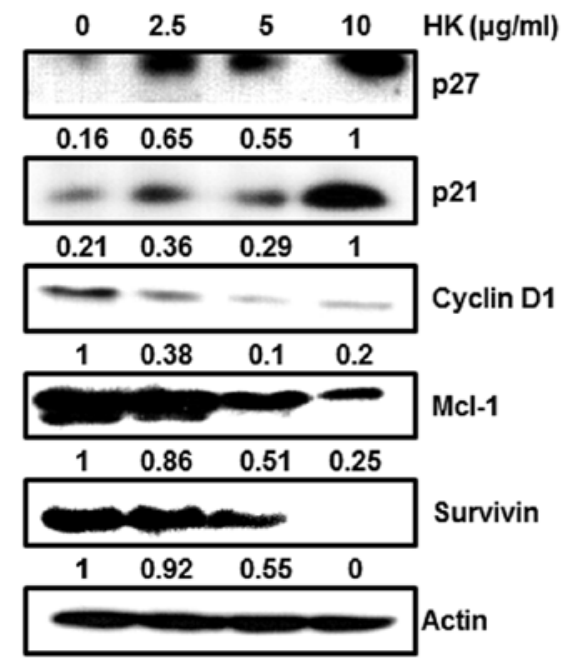

Figure 4. The effect of honokiol (HK) on downstream target protein of specificity protein 1 (Sp1). (A) HN-22 and (B) HSC-4 cells were incubated with HK (2.5, 5 and $10 \mu \mathrm{g} / \mathrm{ml}$ ) or DMSO for $48 \mathrm{~h}$. The protein expressions of p27, p21, cyclin D1, Mcl-1 and survivin were analyzed by western blot analysis against a specific antibody. Forty microgram of cellular extract per lane was separated on SDS-PAGE gel as described in Materials and methods. Equal loading of proteins was confirmed by blotting of anti-actin antibody. The values measured by Image J densitometry are representative of two or three independent experiments.
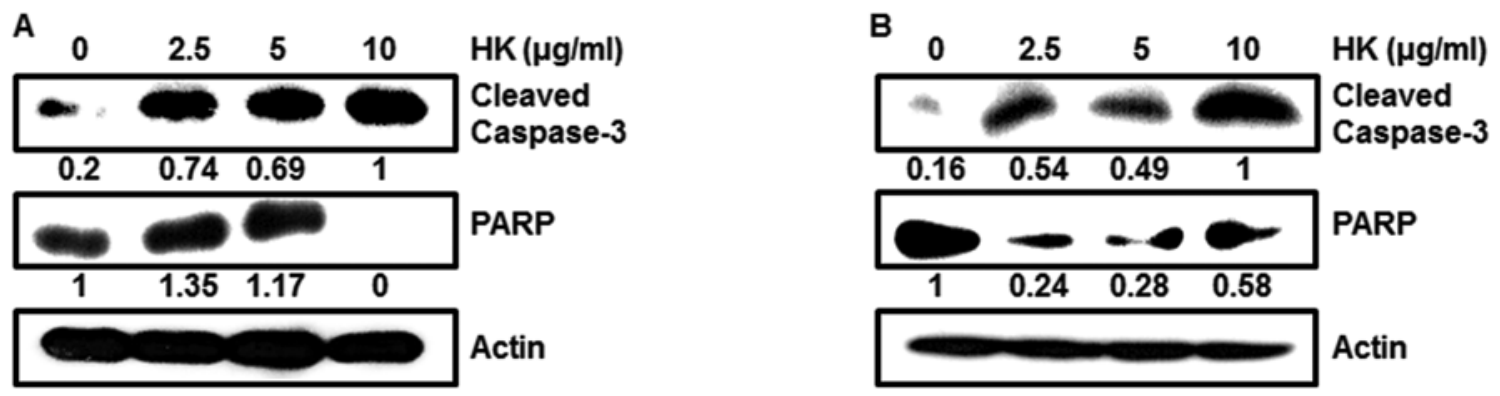

Figure 5. The effect of honokiol (HK) on apoptosis of OSCCs. (A) HN-22 and (B) HSC-4 cells were treated with or without the indicated concentrations of HK $(0,2.5,5$ and $10 \mu \mathrm{g} / \mathrm{ml})$ for $48 \mathrm{~h}$. The cell lysates were determined by western blot analysis with anti-cleaved-caspase-3 and anti-PARP antibodies. Equal loading of proteins was confirmed by blotting of anti-actin antibody. The values measured by Image $\mathrm{J}$ densitometry are representative of three independent experiments.

Transcription factor, Sp1 is known to be ubiquitously expressed and closely associated in various cellular processes through its anti-tumor activity and regulation of signal transductions (19). Interestingly, many different types of cancer cells were reported to show highly enhanced Sp1 expression levels $(24,25,28)$. Therefore, numerous studies have investigated whether up-regulated Sp1 could have an effect on biological processes such as proliferation, differentiation and oncogenesis (19). As an example to cell cycle progression, down-regulation of Sp1 level by siSpl, decoy or ectopic expression of dominantnegative protein induced $G_{1}$ phase cell cycle arrest in human glioblastoma, lung, pancreatic and cervical cancer cells, resulting from alterations of cycle modulating proteins such as cyclin D1 and p27 $(22,23,29,30)$. Therefore, $\mathrm{Sp1}$ has been suggested as an ideal target for molecular therapy against cancer. Our results show that Sp1 was significantly reduced in the HK treated cells (Fig. 2) and pro-apoptotic proteins, PARP and caspase-3, were also regulated toward apoptosis (Fig. 5). These cellular effects of HK were similar to the effects produced by the Sp1 specific inhibitor, mithramycin A which inhibited the expres- sion and transcriptional regulatory activity of Sp1 on target genes including $c-m y c$, cyclin D1, Mcl-1 and survivin (26,27,31). To evaluate whether HK can change target protein expression levels and apoptosis related proteins in OSCCs, we monitored the proteins whose expression was closely associated with cell cycle arrest and survival, to gain mechanistic insights into the role of anticancer effect in OSCCs. Moreover, expression levels of transcriptional regulatory factors of cyclin-dependent kinase inhibitors (CKI), p27 and p21, were studied extensively. Many human cancers frequently show down-regulation of p27 which is correlated with cancer cell malignancy (32). Another cdk inhibitor, p21, is also down-regulated in various human cancers including colorectal, tonsillar carcinoma, gastric and breast cancer (33). Both p21 and p27 are well characterized as negative regulators of cell cycle progression and their functional roles in $\mathrm{G}_{1}$ phase arrest result from the interaction of cyclins and cyclin-dependent kinase (CDKs) complexes $(34,35)$. Therefore, we postulated that if CDKs are positively regulated in cells by a therapeutic agent, malignancies of cancer cells could be effectively suppressed via inhibition of cell cycle arrest. In this 
study, we found that two CKIs, p21 and p27, were significantly increased in $\mathrm{HK}$ dose-dependent manner, whereas their upstream regulator Sp1 was found to be decreased (Fig. 4). These results suggest that $\mathrm{HK}$ was able to negatively regulate $\mathrm{Sp} 1$ expression, resulting in down-regulation of $\mathrm{p} 21$ and $\mathrm{p} 27$.

Another cell cycle involving protein, cyclin D1, was also regulated by $\mathrm{HK}$ treatement. It has been reported that cyclin D1 is indispensable for cell cycle progression because it promotes $\mathrm{G}_{1} / \mathrm{S}$ phase transition via interaction with cyclin dependent kinases. Thus, its expression level is closely associated with tumorigenesis and cell maintenance. A study showed that cyclin D1 was induced by oncogenes including Ras, Src and $\beta$-catenin, when cells were stimulated by oncogenic signals (36-38). Also, an increased level of cyclin D1 has been frequently observed in human cancers (39). Therefore, it is likely that reduction of Spl by HK treatment could not induce transcriptional activation of cyclin D1 on the promoter, resulting in suppression of neoplastic proliferation of OSCCs.

Unlike the cell cycle arrest proteins such as p21 and p27, we observed that pro-survival proteins were significantly reduced by HK in OSCCs. It is known that survivin is a member of inhibitor of apoptosis protein family and its expression level has been considered to play an important role in oncogenesis (40). Numerous studies have demonstrated that negative regulation of survivin expression or inhibition of its cellular function could lead to apoptotic cell death in cancer cells (40). Recently, a study reported that the transcription factor Sp1 can regulate transactivation of survivin via direct binding to the GC-rich promoter region $(41,42)$. Another study revealed that anti-apoptotic protein, Mcl-1, is a member of the Bcl-2 family and is also associated in cancer progression and malignancies (43-45). The down-regulation of Mcl-1 and survivin promotes apoptosis in cancer cells (46-48). Based on these reports, modulation of survivin and Mcl-1 could effectively suppress oncogenesis in vivo and in vitro, suggesting the potential use of these proteins in cancer treatment as a potential therapeutic target gene. To further confirm whether HK could modulate anti-apoptotic protein expressions toward apoptosis, we monitored alterations of Mcl-1 and survivin when cells were treated with different doses. Mcl-1, survivin and cell cycle regulatory proteins were greatly reduced by HK treatment in a dose-dependent manner (Fig. 4). Therefore, HK can positively regulate p27 and p21, and negatively regulate cyclin D1, Mcl-1 and survivin in OSCCs, resulting in activation of a caspase-dependent apoptosis pathway through activated caspase-3 and PARP (Fig. 5).

In this study, we investigated the cancer chemoprevention effect of HK on OSCCs. Our results indicate that HK has cell growth inhibitory activity and induces apoptosis in OSCCs through inhibition of Sp1 expression, followed by transcriptional regulation of the cell cycle regulating and anti-apoptotic proteins. Taken together, HK might be a promising therapeutic agent in the treatment of oral cancers. However, molecular mechanisms and clinical studies for $\mathrm{HK}$ are necessary to elucidate its unexpected potential toxicity and its clinical applications.

\section{Acknowledgements}

This research was supported by Basic Science Research program through the National Research Foundation Korea (NRF) Funded by the Ministry of Education, Science and Technology (2011-
0008463 and 2010-0021532) and the Cooperative Research Program for Agriculture Science and Technology Development (PJ007963), Rural Development Administration, Republic of Korea.

\section{References}

1. Hamada T, Wakamatsu T, Miyahara M, et al: MUC4: a novel prognostic factor of oral squamous cell carcinoma. Int J Cancer 130: 1768-1776, 2012.

2. Warnakulasuriya S: Global epidemiology of oral and oropharyngeal cancer. Oral Oncol 45: 309-316, 2009.

3. Newman DJ and Cragg GM: Natural products as sources of new drugs over the 30 years from 1981 to 2010 . J Nat Prod 75: 311-335, 2012.

4. Fried LE and Arbiser JL: Honokiol, a multifunctional antiangiogenic and antitumor agent. Antioxid Redox Signal 11: 1139-1148, 2009

5. Fujita M, Itokawa $\mathrm{H}$ and Sashida Y: Studies on the components of Magnolia obovata Thunb. 3. Occurrence of magnolol and honokiol in M. obovata and other allied plants. Yakugaku Zasshi 93: 429-434, 1973 (In Japanese).

6. Teng CM, Chen CC, Ko FN, et al: Two antiplatelet agents from Magnolia officinalis. Thromb Res 50: 757-765, 1988.

7. Liou KT, Lin SM, Huang SS, Chih CL and Tsai SK: Honokiol ameliorates cerebral infarction from ischemia-reperfusion injury in rats. Planta Med 69: 130-134, 2003.

8. Lo YC, Teng CM, Chen CF, Chen CC and Hong CY: Magnolol and honokiol isolated from Magnolia officinalis protect rat heart mitochondria against lipid peroxidation. Biochem Pharmacol 47: 549-553, 1994.

9. Kuribara H, Kishi E, Hattori N, Yuzurihara M and Maruyama Y: Application of the elevated plus-maze test in mice for evaluation of the content of honokiol in water extracts of magnolia. Phytother Res 13: 593-596, 1999.

10. Battle TE, Arbiser J and Frank DA: The natural product honokiol induces caspase-dependent apoptosis in B-cell chronic lymphocytic leukemia (B-CLL) cells. Blood 106: 690-697, 2005.

11. Shigemura K, Arbiser JL, Sun SY, et al: Honokiol, a natural plant product, inhibits the bone metastatic growth of human prostate cancer cells. Cancer 109: 1279-1289, 2007.

12. Garcia A, Zheng Y, Zhao C, et al: Honokiol suppresses survival signals mediated by Ras-dependent phospholipase D activity in human cancer cells. Clin Cancer Res 14: 4267-4274, 2008.

13. Deng J, Qian Y, Geng L, et al: Involvement of p38 mitogen-activated protein kinase pathway in honokiol-induced apoptosis in a human hepatoma cell line (hepG2). Liver Int 28: 1458-1464, 2008.

14. Li Z, Liu Y, Zhao X, et al: Honokiol, a natural therapeutic candidate, induces apoptosis and inhibits angiogenesis of ovarian tumor cells. Eur J Obstet Gynecol Reprod Biol 140: 95-102, 2008.

15. Bai X, Cerimele F, Ushio-Fukai M, et al: Honokiol, a small molecular weight natural product, inhibits angiogenesis in vitro and tumor growth in vivo. J Biol Chem 278: 35501-35507, 2003.

16. Sheu ML, Chiang CK, Tsai KS, et al: Inhibition of NADPH oxidase-related oxidative stress-triggered signaling by honokiol suppresses high glucose-induced human endothelial cell apoptosis. Free Radic Biol Med 44: 2043-2050, 2008.

17. Lee J, Jung E, Park J, et al: Anti-inflammatory effects of magnolol and honokiol are mediated through inhibition of the downstream pathway of MEKK-1 in NF-kappaB activation signaling. Planta Med 71: 338-343, 2005.

18. Tse AK, Wan CK, Shen XL, Yang M and Fong WF: Honokiol inhibits TNF-alpha-stimulated NF-kappaB activation and NF-kappaB-regulated gene expression through suppression of IKK activation. Biochem Pharmacol 70: 1443-1457, 2005.

19. Li L and Davie JR: The role of Sp1 and Sp3 in normal and cancer cell biology. Ann Anat 192: 275-283, 2010.

20. Hirano T, Gotoh M and Oka K: Natural flavonoids and lignans are potent cytostatic agents against human leukemic HL-60 cells. Life Sci 55: 1061-1069, 1994.

21. Yang SE, Hsieh MT, Tsai TH and Hsu SL: Down-modulation of Bcl-XL, release of cytochrome $\mathrm{c}$ and sequential activation of caspases during honokiol-induced apoptosis in human squamous lung cancer $\mathrm{CH} 27$ cells. Biochem Pharmacol 63: $1641-1651,2002$. 
22. Abdelrahim M, Smith R III, Burghardt R and Safe S: Role of Sp proteins in regulation of vascular endothelial growth factor expression and proliferation of pancreatic cancer cells. Cancer Res 64: 6740-6749, 2004

23. Ishibashi H, Nakagawa $\mathrm{K}$, Onimaru $\mathrm{M}$, et al: $\mathrm{Sp1}$ decoy transfected to carcinoma cells suppresses the expression of vascular endothelial growth factor, transforming growth factor beta1, and tissue factor and also cell growth and invasion activities. Cancer Res 60: 6531-6536, 2000.

24. Kong LM, Liao CG, Fei F, Guo X, Xing JL and Chen ZN: Transcription factor Sp1 regulates expression of cancer-associated molecule CD147 in human lung cancer. Cancer Sci 101: 1463-1470, 2010.

25. Chuang JY, Wu CH, Lai MD, Chang WC and Hung JJ: Overexpression of Sp1 leads to p53-dependent apoptosis in cancer cells. Int J Cancer 125: 2066-2076, 2009.

26. Choi ES, Shim JH, Jung JY, et al: Apoptotic effect of tolfenamic acid in androgen receptor-independent prostate cancer cell and xenograft tumor through specificity protein 1 . Cancer Sci 102: 742-748, 2011.

27. Shim JH, Shin JA, Jung JY, et al: Chemopreventive effect of tolfenamic acid on KB human cervical cancer cells and tumor xenograft by downregulating specificity protein 1 . Eur J Cancer Prev 20: 102-111, 2011.

28. Davie JR, He S, Li L, et al: Nuclear organization and chromatin dynamics - Sp1, Sp3 and histone deacetylases. Adv Enzyme Regul 48: 189-208, 2008.

29. Grinstein E, Jundt F, Weinert I, Wernet P and Royer HD: Sp1 as G1 cell cycle phase specific transcription factor in epithelial cells. Oncogene 21: 1485-1492, 2002.

30. Chen F, Zhang F, Rao J and Studzinski GP: Ectopic expression of truncated Sp1 transcription factor prolongs the $S$ phase and reduces the growth rate. Anticancer Res 20: 661-667, 2000.

31. Blume SW, Snyder RC, Ray R, Thomas S, Koller CA and Miller DM: Mithramycin inhibits SP1 binding and selectively inhibits transcriptional activity of the dihydrofolate reductase gene in vitro and in vivo. J Clin Invest 88: 1613-1621, 1991.

32. Porter PL, Malone KE, Heagerty PJ, et al: Expression of cell-cycle regulators p27Kip1 and cyclin E, alone and in combination, correlate with survival in young breast cancer patients. Nat Med 3: 222-225, 1997.

33. Abbas T and Dutta A: p21 in cancer: intricate networks and multiple activities. Nat Rev Cancer 9: 400-414, 2009.

34. Sherr CJ and Roberts JM: CDK inhibitors: positive and negative regulators of G1-phase progression. Genes Dev 13: 1501-1512, 1999.

35. Murray AW: Recycling the cell cycle: cyclins revisited. Cell 116: $221-234,2004$
36. Albanese C, Johnson J, Watanabe G, et al: Transforming p21 ras mutants and c-Ets-2 activate the cyclin D1 promoter through distinguishable regions. J Biol Chem 270: 23589-23597, 1995.

37. Lee RJ, Albanese C, Stenger RJ, et al: pp60(v-src) induction of cyclin D1 requires collaborative interactions between the extracellular signal-regulated kinase, p38, and Jun kinase pathways. A role for cAMP response element-binding protein and activating transcription factor- 2 in pp60( $\mathrm{v}$-src) signaling in breast cancer cells. J Biol Chem 274: 7341-7350, 1999.

38. Shtutman M, Zhurinsky J, Simcha I, et al: The cyclin D1 gene is a target of the beta-catenin/LEF-1 pathway. Proc Natl Acad Sci USA 96: 5522-5527, 1999.

39. Weinstein IB: Relevance of cyclin D1 and other molecular markers to cancer chemoprevention. J Cell Biochem (Suppl) 25: 23-28, 1996.

40. Li F: Survivin study: what is the next wave? J Cell Physiol 197: 8-29, 2003.

41. Xu R, Zhang P, Huang J, Ge S, Lu J and Qian G: Sp1 and Sp3 regulate basal transcription of the survivin gene. Biochem Biophys Res Commun 356: 286-292, 2007.

42. Chun JY, Hu Y, Pinder E, Wu J, Li F and Gao AC: Selenium inhibition of survivin expression by preventing Sp1 binding to its promoter. Mol Cancer Ther 6: 2572-2580, 2007.

43. Yoon JH, Werneburg NW, Higuchi H, et al: Bile acids inhibit Mcl-1 protein turnover via an epidermal growth factor receptor/Raf-1-dependent mechanism. Cancer Res 62: 6500-6505, 2002.

44. Okaro AC, Deery AR, Hutchins RR and Davidson BR: The expression of antiapoptotic proteins Bcl-2, Bcl-X(L), and Mcl-1 in benign, dysplastic, and malignant biliary epithelium. J Clin Pathol 54: 927-932, 2001

45. Akgul C: Mcl-1 is a potential therapeutic target in multiple types of cancer. Cell Mol Life Sci 66: 1326-1336, 2009.

46. Andersson Y, Juell S and Fodstad O: Downregulation of the antiapoptotic MCL-1 protein and apoptosis in MA-11 breast cancer cells induced by an anti-epidermal growth factor receptor-pseudomonas exotoxin a immunotoxin. Int $\mathrm{J}$ Cancer 112: 475-483, 2004

47. Chetoui N, Sylla K, Gagnon-Houde JV, et al: Down-regulation of mcl-1 by small interfering RNA sensitizes resistant melanoma cells to fas-mediated apoptosis. Mol Cancer Res 6: 42-52, 2008

48. Wei SH, Dong K, Lin F, et al: Inducing apoptosis and enhancing chemosensitivity to gemcitabine via RNA interference targeting Mcl-1 gene in pancreatic carcinoma cell. Cancer Chemother Pharmacol 62: 1055-1064, 2008. 